\title{
The Impact of Lifetime Alcohol and Cigarette Smoking Loads on Multiple Sclerosis Severity
}

\begin{abstract}
Andrei Ivashynka ${ }^{1,2 *}$, Massimiliano Copetti ${ }^{3}$, Paola Naldi ${ }^{4}$, Sandra $D^{\prime}$ Alfonso ${ }^{1,5}$ and Maurizio A. Leone ${ }^{2,5}$

${ }^{1}$ Department of Health Sciences, University of Eastern Piedmont, Novara, Italy, ${ }^{2}$ Neurology Unit, Department of Medical Sciences, Fondazione IRCCS Casa Sollievo della Sofferenza, San Giovanni Rotondo, Italy, ${ }^{3}$ Unit of Biostatistics, Fondazione IRCCS Casa Sollievo della Sofferenza, San Giovanni Rotondo, Italy, ${ }^{4}$ Department of Neurology, University Hospital "Maggiore della Carità", Novara, Italy, ${ }^{5}$ Interdisciplinary Research Center of Autoimmune Diseases, University of Eastern Piedmont, Novara, Italy
\end{abstract}

Background: The association between lifestyle factors and Multiple Sclerosis (MS) disease severity and progression has been investigated to a lesser extent compared with susceptibility to the disease.

Objective: We aimed to assess the impact of lifetime alcohol and cigarette smoking load on MS severity.

Methods: Design: a cross-sectional study. Three hundred fifty-one patients consecutively admitted to the Department of Neurology were asked to complete the "Questionnaire of Lifestyle" (part of the European Prospective Investigation into Cancer and Nutrition project). An estimation of the cumulative lifetime cigarette smoking and alcohol load was calculated as the weighted sum of the mean number of cigarettes smoked and standard alcoholic drinks consumed per day at different ages. The measure of exposure was expressed in terms of pack-year and drink-year. Disease severity was estimated by the Multiple Sclerosis Severity Score (MSSS). Logistic regression analyses were performed using MSSS (first tertile vs. third tertile) as the outcome.

Results: The median MSSS was higher (3.2 vs. 2.3, $p=0.002)$ in ever- vs. never-smokers, but we did not find a difference between ever- and never-drinkers (2.7 vs. $2.8, p=n s)$. Ever-smokers were almost twice as likely to fall in the upper MSSS tertile than never-smokers. Ever-drinkers did not show a statistically significant association between alcohol intake and MS severity. The risk of falling in the worst MSSS tertile for smokers was $10.81(2.0-58.48 ; p<0.01)$ if they were never-drinkers, whereas it was only 1.65 $(0.89-3.03, p=0.11)$ if they were also drinkers. On the other side, the risk of falling in the worst MSSS tertile for drinkers did not change as much, whether they also were smokers (0.46; 0.13-1.65; $p=0.23$ ) or not (1.49; 0.55-4.04, $p=0.43)$.

Conclusions: Cigarette smoking, unlike alcohol consumption, is associated with MS severity. Alcohol consumption may attenuate the effect of smoking on disease severity, acting as an effect modifier. The biological background of this effect is unknown. The limitations of our study are mostly due to its cross-sectional design.

Keywords: multiple sclerosis, multiple sclerosis severity score, lifetime alcohol load, lifetime cigarette smoking load, risk factors, cross-sectional study 


\section{INTRODUCTION}

Multiple sclerosis (MS) is an autoimmune disease of the central nervous system with a heterogeneous clinical course, characterized by multifocal inflammatory demyelination and secondary axonal degeneration, in which the interaction between environmental and genetic protective and risk factors leads to the chronic activation of immune cells, and neuronal injury $(1,2)$.

There is ample evidence that lifestyle habits, such as alcohol use and cigarette smoking, are risk factors for the development of autoimmune diseases. Cigarette smoking has been associated with susceptibility to MS (3) as well as to other autoimmune diseases (4), whereas the effect of alcohol is still controversial (5). The association of lifestyle factors with MS disease severity and progression is less explored compared with susceptibility to the disease.

Potentially modifiable lifestyle factors, such as alcohol consumption and cigarette smoking might play a role in MS progression, and this is essential from a clinical as well as an etiological point of view. In some studies the harmful effect of cigarette smoking on the course of already established MS was proved (6-9), whereas only a handle of studies had evaluated the relationship between alcohol intake and disease severity so far $(5,9,10)$, and none of them had studied the combined effect of these two factors.

Since both alcohol use and cigarette smoking are common chronic risk factors, their long-term effects may depend on cumulative lifetime exposure, even in low to moderate doses. That is why the study goal was to evaluate the association between lifetime alcohol consumption and lifetime cigarette smoking with MS severity.

\section{METHODS}

\section{Patients and Data Collection}

The research was designed as a cross-sectional study. It was approved by the Ethics Committee of the University Hospital "Maggiore della Carità" in Novara (Italy). Patients followed at the MS Center of the Department of Neurology were consecutively recruited between 2011 and 2012. It is a first-referral MS Center, covering a catchment area of around 400,000 populations. MS patients are referred by other neurologists and General Practitioners and are regularly followed with twice yearly visits. Of the 356 patients who were asked to participate, only five refused to take part in the study. Written informed consent was obtained from 351 participants. All cases were diagnosed by neurologists according to the McDonald criteria (11). Patients were enrolled during follow-up visits scheduled out of a relapse. Demographics (date of birth, age, sex, profession, marital status, education) and clinical data (disease course, date of onset and diagnosis, EDSS) were collected. Cigarette smoking and alcohol consumption histories were evaluated with the "Questionnaire of Lifestyle" which is part of the European Prospective Investigation into Cancer and Nutrition project (EPIC) study (12). All the patients were asked to fill the questionnaire during the clinic visit. In case of difficulty, they were free to ask for help one interviewer who was blinded to the clinical history and severity evaluation.

\section{Disease Severity Examination}

Disease severity was estimated through the Expanded Disability Status Scale (EDSS) and the Multiple Sclerosis Severity Score (MSSS). The MSSS corrects EDSS for disease duration, allowing to compare an individual's disability with the distribution of scores in cases having similar EDSS scores. The MSSS score (range 0-9.9) was calculated, according to Roxburgh et al. (13).

\section{Exposure Assessment}

Cigarette smoking and alcohol consumption data were collected at recruitment. All subjects were asked to report their cigarette smoking status at the time of the interview (never, former, or current smoker). Never-smokers were patients who had smoked $<100$ cigarettes up to the time of the interview (14). Former smokers were patients who had smoked $>100$ cigarettes and had stopped smoking at least 6 months before the time of the interview. Former smokers were asked to state the age when they started and then quit smoking. Current smokers were those who had smoked $>100$ cigarettes and were still smoking at the time of the interview. Ever-smokers (former or current) were asked to quantify the number of cigarettes per day smoked at recruitment and four age periods $(20,30,40$, and 50 years). For each age period, we calculated the mean number of cigarettes smoked per day based on the questionnaire information and the number of years spent smoking. The cigarette smoking duration (years) was calculated as the difference between age at recruitment (for current smokers) or age at cigarette smoking cessation (for former smokers), and age at start smoking.

An estimation of the cumulative lifetime smoking load was calculated as the weighted sum of the mean number of cigarettes that were smoked per day at different ages, including at the time of recruitment, with weights equal to the cigarette smoking duration for each age period. The measure of exposure was expressed in pack-year, defining a pack as containing 20 cigarettes.

Similarly, detailed information was obtained regarding alcohol consumption during different age periods up to the participants' current age. The alcohol drinking status at the time of the interview (never, former, or current drinker) was assessed. We defined as never-drinkers those who had drunk less than one standard alcoholic drink/month. Former drinkers were patients who had drunk one or more standard alcoholic drinks/month and had stopped drinking at least 6 months before the interview. Ever-drinkers (former or current) were asked to state the number of alcoholic drinks per day by type of beverage (wine, beer and spirits), at the time of interview and four age periods $(20,30,40$, and 50 years). The method used to calculate the cigarette smoking duration was also used to calculate the number of years of alcohol consumption. An Italian standard alcoholic drink (standard alcoholic unit) contains approximately 12 grams of pure ethanol (15), corresponding to a small glass of wine $(125 \mathrm{ml})$, a can of beer $(330 \mathrm{ml})$, or a shot of spirits $(40 \mathrm{ml})$.

In analogy with the cumulative lifetime smoking load, a cumulative lifetime alcohol drinking load (drink-year) was calculated as the weighted sum of the mean number of standard alcoholic units per day at different age periods with weights equal 
to the number of years spent drinking for each age period for each type of beverage, and in total (aggregating all kinds of drinks).

\section{Statistical Analysis}

Patients' baseline characteristics are reported as median with range and frequencies for continuous and categorical variables, respectively. According to their smoking and drinking status at the time of the interview, subjects were classified into never, former or current smokers/drinkers. Ever-(former and current) smokers/ever-drinkers were categorized into two groups (lower load vs. higher load) according to the median value of the packyear or drink-year distribution.

A new categorical variable was defined to explore the combination of smoking and drinking habits. The four categories were: (1) never-smokers/never-drinkers; (2) neversmokers/ever-drinkers; (3) ever-smokers/ever-drinkers; (4) eversmokers/never-drinkers.

MSSS was converted into a trichotomous categorical variable, based on tertiles of the distribution, and logistic regression analyses were performed using MSSS $<1.8$ (first tertile) vs. $>3.9$ (third tertile) as the outcome, thus comparing the two extremes of the MSSS distribution.

Normal distribution assumption was checked using the Shapiro-Wilks test and the Q-Q plot. Comparisons between two or more groups were tested using the Chi-square test for categorical variables and Student's T-test or ANOVA analysis for continuous variables. Non-parametric Mann-Whitney and Kruskal-Wallis tests were used when appropriate.

The association between smoking and drinking habits and disease severity was evaluated using a univariable and multivariable logistic regression model that was adjusted for age, sex, and education. Risks were reported as odds ratios (OR) along with their 95\% confidence intervals (CI). A sensitivity analysis was conducted considering the three types of beverages (wine, beer, and spirits) separately.

To assess the possibility of an effect modification, we evaluated the risk for ever-smokers of being in the upper (worst) MSSS tertile in the two subsets of ever-drinkers and never-drinkers, separately; on the other side, we assessed the risk of ever-drinkers of being in the worst MSSS tertile in the two subgroups of ever-smokers and never-smokers.

A $p$-value $<0.05$ was considered as statistically significant. Analyses were performed using SAS ${ }^{\circledR}$, version 9.3 (SAS Institute, Cary, NC).

\section{RESULTS}

We recruited 351 patients, 235 women and 116 men, with a sex ratio of 2:1. The mean age was $33.0 \pm 10.1$ years at MS onset, and $44.1 \pm 11.7$ years at recruitment. The mean disease duration at recruitment was $11.1 \pm 8.1$ years. The other demographic and clinical characteristics are presented in Table $\mathbf{1 .}$ Only three patients began to smoke after being diagnosed with MS, and 26 patients began to consume alcohol after the MS diagnosis.

Table 2 shows unadjusted comparisons between never and ever-smokers. Ever-smokers were older, more frequently males
TABLE 1 | Demographical, clinical and exposure characteristics of the MS sample.

\begin{tabular}{|c|c|c|}
\hline Variable & Category & $\begin{array}{l}\text { Median (min-max) or } \\
\text { frequency }(\%)\end{array}$ \\
\hline$N$ & & 351 \\
\hline Age at MS onset (years) & & 32 (6.82-64.35) \\
\hline Education (years) & & 13.00 (5.00-24.00) \\
\hline $\begin{array}{l}\text { Disease duration } \\
\text { (years) }\end{array}$ & & $10.00(1.00-48.00)$ \\
\hline EDSS & & $2.00(0.00-8.00)$ \\
\hline MSSS & & $2.70(0.05-9.86)$ \\
\hline \multirow[t]{2}{*}{ The clinical form of MS } & $\begin{array}{l}\text { Relapsing- } \\
\text { remitting }\end{array}$ & $266(75.78)$ \\
\hline & $\begin{array}{l}\text { Progressive } \\
\text { (primary and } \\
\text { secondary) }\end{array}$ & $85(24.22)$ \\
\hline \multicolumn{3}{|l|}{ Cigarette smoking } \\
\hline \multirow[t]{3}{*}{ status } & Current Smokers & $92(26.21)$ \\
\hline & Never-smokers & $161(45.87)$ \\
\hline & Former smokers & 98 (27.92) \\
\hline $\begin{array}{l}\text { Lifetime Cigarette } \\
\text { Smoking Load } \\
\text { (pack-year)* }^{*}\end{array}$ & & $10.63(0.10-78.37)$ \\
\hline \multirow[t]{2}{*}{$\begin{array}{l}\text { Alcohol drinking } \\
\text { status(general) }\end{array}$} & $\begin{array}{l}\text { Current drinker } \\
\text { Never-drinker }\end{array}$ & $\begin{array}{l}256(72.93) \\
68(19.37)\end{array}$ \\
\hline & Former drinker & 27 (7.69) \\
\hline \multirow[t]{3}{*}{ Wine } & Current drinker & $218(62.11)$ \\
\hline & Never-drinker & $108(30.77)$ \\
\hline & Former drinker & $25(7.12)$ \\
\hline \multirow[t]{3}{*}{ Beer } & Current drinker & $181(51.57)$ \\
\hline & Never-drinker & 134 (38.18) \\
\hline & Former drinker & $36(10.26)$ \\
\hline \multirow[t]{3}{*}{ Spirits } & Current drinker & 125 (35.61) \\
\hline & Never-drinker & 165 (47.01) \\
\hline & Former drinker & $61(17.38)$ \\
\hline $\begin{array}{l}\text { Lifetime Alcohol } \\
\text { Load(drink-year) }^{\star \star}\end{array}$ & & 7.49 (0.36-394.09) \\
\hline
\end{tabular}

*Ever-(current and former) smokers $(n=190)$.

${ }^{* *}$ Ever-(current and former) drinkers $(n=283)$.

and had a lower education level than never-smokers. As expected, ever-smokers tend to drink more than people who had never smoked. Finally, the univariable analyses showed that the disease, in term of MSSS, was more severe in people who had smoked than in never-smokers. The association between MSSS and smoking remained significant in the subsample of drinkers (data not shown, $p=0.037$ ). No significant differences in disease severity were found between smokers with lower and higher smoke loads.

Table 3 shows unadjusted comparisons between never and ever-drinkers. As expected, alcohol consumption was more frequent in males than in females. No difference in disease severity based on MSSS scores was noted when comparing ever with never-drinkers. MSSS was significantly lower for patients with lower alcohol load than for those with higher alcohol load. The association between MSSS and alcohol load remained 
TABLE 2 | Subgroup comparisons according to cigarette smoking status.

\begin{tabular}{|c|c|c|c|c|c|c|c|}
\hline Variable & Category & Never-smokers & Ever-smokers & $p$-value & $\begin{array}{c}\text { Lower } \\
\text { smoke } \\
\text { load* }^{*}\end{array}$ & $\begin{array}{c}\text { Higher } \\
\text { smoke } \\
\text { load* }^{*}\end{array}$ & $p$-value \\
\hline \multirow[t]{2}{*}{$N$} & & 161 & 190 & & 95 & 95 & \\
\hline & & \multicolumn{2}{|c|}{ Median (min-max) or frequency $(\%)$} & & \multicolumn{2}{|c|}{ Median (min-max) or frequency $(\%)$} & \\
\hline $\begin{array}{l}\text { Age at MS onset } \\
\text { (years) }\end{array}$ & & $\begin{array}{c}30.11 \\
(6.82-64.35)\end{array}$ & $\begin{array}{c}34.19 \\
(12.21-60.70)\end{array}$ & 0.005 & $\begin{array}{c}31.53 \\
(12.21-60.70)\end{array}$ & $\begin{array}{c}35.95 \\
(12.76-60.70)\end{array}$ & 0.013 \\
\hline Education (years) & & $\begin{array}{c}13.00 \\
(5.00-24.00)\end{array}$ & $\begin{array}{c}11.00 \\
(5.00-23.00)\end{array}$ & 0.001 & $\begin{array}{c}12.00 \\
(5.00-23.00)\end{array}$ & $\begin{array}{c}11.00 \\
(5.00-19.00)\end{array}$ & 0.044 \\
\hline \multirow[t]{2}{*}{ Sex } & Female & $120(74.53)$ & $115(60.53)$ & 0.005 & $67(70.53)$ & $48(50.53)$ & 0.005 \\
\hline & Male & $41(25.47)$ & $75(39.47)$ & & $28(29.47)$ & $47(49.47)$ & \\
\hline $\begin{array}{l}\text { Disease duration } \\
\text { (years) }\end{array}$ & & $\begin{array}{c}10.00 \\
(1.00-48.00)\end{array}$ & $9.00(1.00-41.00)$ & 0.586 & $\begin{array}{c}8.00 \\
(1.00-33.00)\end{array}$ & $\begin{array}{c}11.00 \\
(1.00-41.00)\end{array}$ & 0.001 \\
\hline MSSS & & $2.33(0.05-9.86)$ & $3.21(0.13-9.68)$ & 0.002 & $\begin{array}{c}3.44 \\
(0.16-9.59)\end{array}$ & $3.10(0.13-9.68)$ & 0.982 \\
\hline \multirow[t]{2}{*}{ Clinical form of MS } & $\begin{array}{l}\text { Relapsing- } \\
\text { remitting }\end{array}$ & $128(79.50)$ & $138(72.63)$ & 0.127 & $76(80.00)$ & $62(65.26)$ & 0.020 \\
\hline & $\begin{array}{l}\text { Progressive } \\
\text { forms }\end{array}$ & $33(20.50)$ & $52(27.37)$ & & $19(20.00)$ & $33(34.74)$ & \\
\hline \multirow[t]{3}{*}{$\begin{array}{l}\text { Alcohol drinking } \\
\text { status }\end{array}$} & $\begin{array}{l}\text { Current } \\
\text { drinker }\end{array}$ & 109 (67.70) & $147(77.37)$ & 0.076 & 77 (81.05) & 70 (73.68) & 0.332 \\
\hline & Never-drinker & 35 (21.74) & 33 (17.37) & & 15 (15.79) & 18 (18.95) & \\
\hline & $\begin{array}{l}\text { Former } \\
\text { drinker }\end{array}$ & $17(10.56)$ & $10(5.26)$ & & $3(3.16)$ & $7(7.37)$ & \\
\hline $\begin{array}{l}\text { Lifetime alcohol } \\
\text { Load (drink-year) }\end{array}$ & & $\begin{array}{c}2.65 \\
(0.00-101.95)\end{array}$ & $\begin{array}{c}8.00 \\
(0.00-394.09)\end{array}$ & $<0.001$ & $\begin{array}{c}6.85 \\
(0.00-86.05)\end{array}$ & $\begin{array}{c}11.47 \\
(0.00-394.09)\end{array}$ & 0.173 \\
\hline
\end{tabular}

"The two groups (lower vs. higher Lifetime Cigarette Smoking Load) were categorized according to their median value.

significant in the sub-sample of smokers (data not shown, $p=$ 0.026). However, the other predictive factors (age, sex, age at onset, smoking) were unequally distributed in the two groups.

The MSSS distribution was compared across the different categories according to drinking/smoking habits (Figure 1). MS severity was lowest in patients who never had drunk nor smoked, highest in patients who had smoked but had never drunk, and intermediate for patients who had only drunk or had both drunk and smoked.

Logistic regression models after controlling for age, sex, and education were estimated to confirm these results, comparing the extremes of the MSSS distribution (upper vs. lower tertile) (Table 4). Ever-smokers were almost twice as likely to fall in the upper than in the lower MSSS tertile compared with never-smokers, whereas no difference was found for drinking habits (model A and B). In both models of ever-smokers and ever-drinkers (model C and D) no significant association was found between categories of lifetime load and disease severity. However, patients who had only smoked were six times more likely to fall in the upper rather than the lower MSSS tertile compared with patients who never had drunk or smoked, but alcohol consumption seems to mitigate this association (model E).

To further explore the combined effect of the two exposures, we stratified patients by drinking and smoking habits, adjusting for sex and age. The risk of falling in the worst MSSS tertile for smokers was $10.81(2.0-58.48 ; p<0.01)$ if they were neverdrinkers, whereas it was only $1.65(0.89-3.03, p=0.11)$ if they were also drinkers. On the other side, the risk of falling in the worst MSSS tertile for drinkers did not change as much, whether they also were smokers $(0.46 ; 0.13-1.65 ; p=0.23)$ or not $(1.49$; $0.55-4.04, p=0.43)$.

\section{DISCUSSION}

The results of this study confirm those of several observational studies suggesting that cigarette smoking may exert an influence on the MS course. Cohort studies of Clinically Isolated Syndrome patients concluded that conversion to clinically definite MS and disease progression were more rapid in ever-smokers compared to never-smokers $(8,16)$. Hernan et al. found that cigarette smoking increased the risk of conversion from relapsingremitting to secondary progressive MS (17). A Swedish study of self-reported data from MS patients found that, after a median of 6 years, ever-smokers were more likely to have progressive disease compared to never-smokers. Moreover, the effects on the rate of conversion to progressive MS and earlier age of progression onset were highest in those who began smoking before the age of 15. Early onset of regular smoking also associated with a higher percentage of primary progressive MS when compared with never-smokers (18). 
TABLE 3 | Subgroup comparisons, according to the alcohol drinking status.

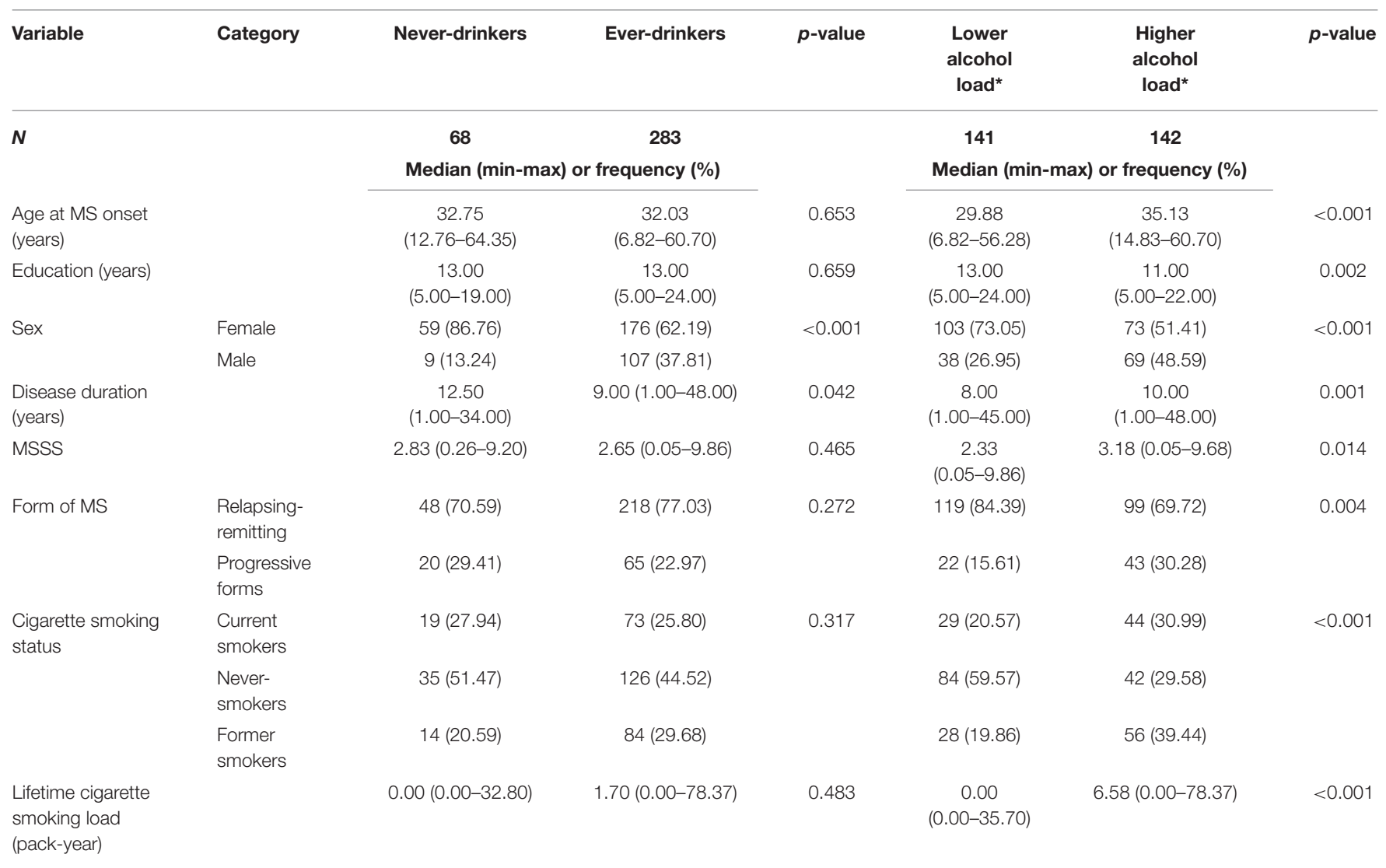

"The two groups (lower vs. higher Lifetime Alcohol Load) were categorized according to their median value.

Our study found no significant difference in MS severity between ever-drinkers and never-drinkers. MSSS values were lower among people who had drunk less alcohol during their lifetime compared to those who had consumed higher amounts of alcohol. To date, only a few studies have evaluated a possible effect of alcohol consumption on disease severity in MS. Alcohol consumption was associated with less total disability in cross-sectional studies that used different outcomes and MS severity end-points: EDSS (19, 20), MSSS (20), PatientDetermined Disease Steps (21), Incapacity Status Scale Scores (22), and reaching EDSS $6(23,24)$. Two studies showed an inverse dose-response association between alcohol consumption and disability measured with EDSS $(19,21)$, while two other cross-sectional studies did not find any association $(25,26)$. In addition to the inherent limitations of the cross-sectional study design, these studies were affected by selection bias, as were enrolled persons from MS patient associations or online, or used convenience samples $(19,22,23)$. Some studies had a low response rate $(19,25)$, and did not take into account possible confounders (age, sex, disease duration, type of therapy, Body Mass Index, other chronic diseases). The only prospective study (9) found that ex-consumers of alcohol had a lower risk of MS progression than current consumers, but after stratifying by sex, no significant differences were found among nonconsumers, ex-consumers and current alcohol consumers. In previous studies, alcohol consumption was ascertained in terms

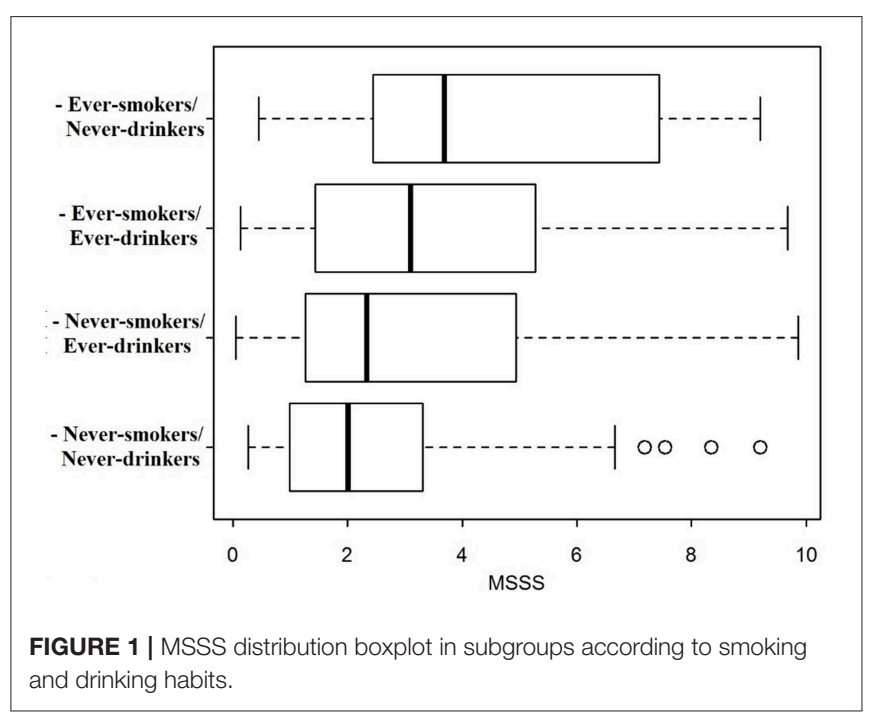

of quantity/frequency $(19,21,23,26)$, duration of consumption (20), or using validated questionnaires $(9,22,25,26)$. No experimental clinical study has been conducted because of ethical limitations.

The most remarkable result of our study is that eversmokers/ever-drinkers MS patients were only twice as likely to 
TABLE 4 | Age, sex, and education-adjusted logistic regression models: upper MSSS tertile vs. lower MSSS tertile ( $N=230)$.

\begin{tabular}{|c|c|c|c|c|c|}
\hline & & & OR & $95 \% \mathrm{Cl}$ & $p$-value \\
\hline Model A & Cigarette smoking status & Ever-smokers $(n=120)$ vs. Never-smokers $(n=110)$ & 1.89 & $1.08-3.30$ & 0.026 \\
\hline Model B & Alcohol drinking status & Ever-drinkers ( $n=190)$ vs. Never-drinkers $(n=40)$ & 0.98 & $0.47-2.03$ & 0.961 \\
\hline \multirow[t]{3}{*}{ Model C } & Lifetime cigarette smoking load (pack-year) $^{\star}$ & Lower LCSL $(n=61)$ & 2.64 & $1.33-5.24$ & 0.005 \\
\hline & & Higher LCSL $(n=59)$ & 1.30 & $0.65-2.61$ & 0.465 \\
\hline & & Never-smokers $(n=110)$ & 1.00 & & \\
\hline \multirow[t]{3}{*}{ Model D } & Lifetime alcohol load (drink-year) ${ }^{\star \star}$ & Lower LAL $(n=87)$ & 0.86 & $0.38-1.92$ & 0.711 \\
\hline & & Higher LAL $(n=103)$ & 1.10 & $0.50-2.42$ & 0.808 \\
\hline & & Never-drinkers $(n=40)$ & 1.00 & & \\
\hline \multirow[t]{4}{*}{ Model E } & Four categories & Never-smokers/ever-drinkers $(n=87)$ & 1.75 & $0.64-4.81$ & 0.278 \\
\hline & & Ever-smokers/Ever-drinkers $(n=103)$ & 2.54 & $0.94-6.88$ & 0.067 \\
\hline & & Ever-smokers/Never-drinkers $(n=17)$ & 6.62 & $1.54-28.33$ & 0.011 \\
\hline & & Never-smokers/Never-drinkers $(n=23)$ & 1.00 & & \\
\hline
\end{tabular}

"Lower vs. higher Lifetime Cigarette Smoking Load (Ever-smokers): OR =2.04; 95\% Cl=0.91-4.57.

${ }^{* *}$ Lower vs. higher Lifetime Alcohol Load (Ever-drinkers): $O R=0.78 ; 95 \% \mathrm{Cl}=0.41-1.49$.

LAL, Lifetime Alcohol Load; LCSL, Lifetime Cigarette Smoking Load.

be in the upper MSSS tertile compared to never-smokers/neverdrinkers, whereas ever-smokers who never drank had a 6-fold chance of being in the same upper (worst) tertile. The median MSSS was also higher in ever-smokers/never-drinkers than in ever-smokers/ever-drinkers. The interplay of the two exposures is intricate: a confounding effect was improbable since, after stratification for drinking habits (not/yes), the OR for eversmokers was higher in the never-drinker stratum compared to the ever-drinker stratus ( 10.8 vs. 1.7$)$. It points to the possibility that alcohol acts as an effect modifier of smoking exposure. Insufficient sample size could explain the partial overlap of the CIs.

A similar observation (alcohol-induced attenuation of the effect of smoking) comes from two large studies on MS susceptibility, in which both incident and prevalent MS cases were asked to report their alcohol habits prior to or close to the time of disease onset compared to age- and sex-matched controls (2). Thus, a possible attenuation of the effect of smoking by alcohol use could occur either before the disease onset or during the disease, as shown for the first time in our study.

The biological background of this effect is unknown, and we can only speculate upon it. An interplay between alcohol use and cigarette smoking could occur at different levels. Firstly, both alcohol and smoke are potential modulators of the gastric and intestinal microbiome (27), which may play a critical role in the development or progression of the disease by modulating the inflammatory process (28). Secondly, since both alcohol and smoke might be able to modulate several components of the immune system, the interaction could happen at this level leading to altered inflammatory responses. Many immunological and inflammatory effects of alcohol are dose-dependent (29). Thirdly, alcohol and its metabolites directly affect the central nervous system. The effect of alcoholic beverages depends on many factors, including the properties of the drinks, the presence of additional components, drinking frequency and patterns, dose, and setting of use. Thus, the balance between harmful effects due to the ethanol itself and possible protective effects due to other substances such as resveratrol or flavonoids varies widely. Therefore, future studies on alcohol use and MS risk or severity are needed to explore also the possible dose-effect of alcohol exposure and should take into account the type of beverage and the total alcohol load. We performed an analysis (data not shown) by kind of alcoholic drink, but the study sample size was not sufficiently powered for such subgroup analysis.

The limitations of this study are mostly due to its crosssectional design, where the outcomes of interest and exposures are carried out at the same point in time and do not indicate the sequence of events, whether exposure determines the severity or vice-versa. For this reason, it is not possible to infer causality. Those with a more severe disease progression might have modified their alcohol and smoke habits, and this might have influenced their report of previous exposures. Furthermore, we cannot exclude a recall bias using lifestyle questionnaires for data collection. Our study did not have enough power for subgroup analyses by sex and types of MS, and we could not evaluate the influence of unmeasured confounders, such as BMI, or vitamin $\mathrm{D}$.

On the other hand, our study does present some strengths. Selection bias was minimized because patients were enrolled at a first-referral Center serving most patients of its catchment area, recruitment was consecutive, and the duration of the recruitment period allowed us to recruit almost all the patients followed-up at the MS Center. Recall bias is unavoidable with this type of study, but patients were unaware of the study hypothesis, questionnaires were self-administered, and the helping interviewer was blinded to neurological status. Using the lifetime alcohol load (drink-year) and cigarette smoking load (pack-year) to measure exposure, we were able to study the lifelong cumulative effect of both exposures and not only the amount of exposure at the time of the interview or immediately before. Also, it was examined the impact of smoke and alcohol on the course of MS simultaneously. 
In conclusion, this study adds to the knowledge of the association of two common harmful lifestyle factors with MS severity, supporting the need for further investigation into their role in disease progression and their possible interaction with other lifestyle factors. Given the many potential confounders and the possibility that different factors act in different stages of the disease, further studies need to be large enough to consider all the potential confounders and differentiate between risk factors for susceptibility to the disease and those predictive for severity and MS progression (1). Cumulative measures, such as packyear and drink-year should be used for estimating the lifetime effect of alcohol use and cigarette smoking on the course of MS. This study results also have practical relevance, actively supporting abstinence from smoking in MS patients, whereas to date, no clear recommendation may be issued for social drinking to patients with MS. Since patients with MS exhibit numerous adverse health behaviors (30), attention to the management of potentially modifiable lifestyle factors for slowing progression in patients with MS is worthwhile.

\section{DATA AVAILABILITY}

The raw data supporting the conclusions of this manuscript will be made available by the authors, without undue reservation, to any qualified researcher.

\section{ETHICS STATEMENT}

The work was approved by the Ethics Committee of the University Hospital Maggiore della Carità, Novara, Italy.This neuroepidemiological research was based on the observational (cross-sectional) study design.

\section{AUTHOR CONTRIBUTIONS}

AI: study design, recruitment, and examination of patients, data collecting, management, and analysis, final approval of the version to be published, drafting the paper, agreement to be accountable for all aspects of the work in ensuring that questions

\section{REFERENCES}

1. Koch MW, Metz LM, Agrawal SM, Yong VW. Environmental factors and their regulation of immunity in multiple sclerosis. J Neurol Sci. (2013) 324:106. doi: 10.1016/j.jns.2012.10.021

2. Hedstrom AK, Hillert J, Olsson T, Alfredsson L. Alcohol as a modifiable lifestyle factor affecting multiple sclerosis risk. JAMA Neurol. (2014) 71:300-5. doi: 10.1001/jamaneurol.2013.5858

3. Degelman ML, Herman KM. Smoking and multiple sclerosis: a systematic review and meta-analysis using the Bradford Hill criteria for causation. Mult Scler Relat Disord. (2017) 17:207-16. doi: 10.1016/j.msard.2017.07.020

4. Costenbader KH, Gay S, Alarcon-Riquelme ME, Iaccarino L, Doria A. Genes, epigenetic regulation and environmental factors: which is the most relevant in developing autoimmune diseases? Autoimmun Rev. (2012) 11:604-9. doi: 10.1016/j.autrev.2011.10.022

5. Hempel S, Graham GD, Fu N, Estrada E, Chen AY, Miake-Lye I, et al. A systematic review of modifiable risk factors in the progression of multiple sclerosis. Mult Scler. (2017) 23:525-33. doi: 10.1177/1352458517690270 related to the accuracy or integrity of any part of the work are appropriately investigated and resolved. MC: study design, data analysis, data interpretation, and writing. PN: acquisition of data for the work (in enrolling the sample of multiple sclerosis patients followed at the Department of Neurology of the Maggiore della Carita hospital, to collect patient-related information and informed consents, to perform disease severity examination. Revised the paper critically for important intellectual content (results of analysis and discussion). Dubble-check the paper organization and the accuracy of the provided information, agree with conclusions. Final approval of the version to be published and decide to be accountable for all aspects of the work in ensuring that questions related to the accuracy of the integrity of any part of the work are appropriately investigated and resolved. SD'A: contribution to the conception of the study, final approval of the version of the manuscript to be published, and revising critically the research and the manuscript. ML: study design, recruitment, data management, and analysis, drafting the paper, final approval of the version to be published, agreement to be accountable for all aspects of the work in ensuring that questions related to the accuracy or integrity of any part of the work are appropriately investigated and resolved.

\section{FUNDING}

The study was supported by the Fondo China e Pino, MaffeoFondazione della Comunità del Novarese, Novara, Italy. The funder of the study had no role in study design, data collection, data analysis, data interpretation, or writing of the report. The corresponding author had full access to all the data in the study and had final responsibility for the decision to submit for publication.

\section{ACKNOWLEDGMENTS}

We wish to thank Prof. Roberto Cantello, Prof. Cristoforo Comi, Dr. Domizia Vecchio, Dr. Olga Raymkulova, Mrs. Monica Graziani, Multiple Sclerosis Center, Maggiore della Carità hospital, Novara who collaborated in the recruitment of patients.

6. Pittas F, Ponsonby AL, Van Der Mei IA, Taylor BV, Blizzard L, Groom P, et al. Smoking is associated with progressive disease course and increased progression in clinical disability in a prospective cohort of people with multiple sclerosis. J Neurol. (2009) 256:577-85. doi: 10.1007/s00415-009-0120-2

7. Zivadinov R, Weinstock-Guttman B, Hashmi K, Abdelrahman N, Stosic M, Dwyer M, et al. Smoking is associated with increased lesion volumes and brain atrophy in multiple sclerosis. Neurology. (2009) 73:504-10. doi: 10.1212/WNL.0b013e3181b2a706

8. Correale J, Farez MF. Smoking worsens multiple sclerosis prognosis: two different pathways are involved. J Neuroimmunol. (2015) 281:23-34. doi: 10.1016/j.jneuroim.2015.03.006

9. Paz-Ballesteros WC, Monterrubio-Flores EA, De Jesus Flores-Rivera J, Corona-Vazquez T, Hernandez-Giron C. Cigarette smoking, alcohol consumption and overweight in multiple sclerosis: disability progression. Arch Med Res. (2017) 48:113-20. doi: 10.1016/j.arcmed.2017.03.002

10. Hempel S, Graham GD, Fu N, Estrada E, Chen AY, Miake-Lye I, et al. A systematic review of the effects of modifiable risk factor interventions 
on the progression of multiple sclerosis. Mult Scler. (2017) 23:513-24. doi: $10.1177 / 1352458517690271$

11. Polman CH, Reingold SC, Banwell B, Clanet M, Cohen JA, Filippi M, et al. Diagnostic criteria for multiple sclerosis: 2010 revisions to the McDonald criteria. Ann Neurol. (2011) 69:292-302. doi: 10.1002/ana. 22366

12. Ferrari P, Jenab M, Norat T, Moskal A, Slimani N, Olsen A, et al. Lifetime and baseline alcohol intake and risk of colon and rectal cancers in the European prospective investigation into cancer and nutrition (EPIC). Int $J$ Cancer. (2007) 121:2065-72. doi: 10.1002/ijc.22966

13. Roxburgh RH, Seaman SR, Masterman T, Hensiek AE, Sawcer SJ, Vukusic S, et al. Multiple sclerosis severity score: using disability and disease duration to rate disease severity. Neurology. (2005) 64:1144-51. doi: 10.1212/01.WNL.0000156155.19270.F8

14. Centers for Disease Control. Cigarette smoking among adults-United States, 2000. Morb Mortal Wkly Rep. (2002) 51:642-5.

15. INRAN. Linee Guida per Una Sana Alimentazione Italiana. (2003). p. 53-63.

16. Di Pauli F, Reindl M, Ehling R, Schautzer F, Gneiss C, Lutterotti A, et al. Smoking is a risk factor for early conversion to clinically definite multiple sclerosis. Mult Scler. (2008) 14:1026-30. doi: 10.1177/13524585080 93679

17. Hernan MA, Jick SS, Logroscino G, Olek MJ, Ascherio A, Jick H. Cigarette smoking and the progression of multiple sclerosis. Brain. (2005) 128:1461-5. doi: 10.1093/brain/awh471

18. Sundstrom P, Nystrom L. Smoking worsens the prognosis in multiple sclerosis. Mult Scler. (2008) 14:1031-5. doi: 10.1177/1352458508093615

19. Goodin DS. Survey of multiple sclerosis in northern California. Northern California MS Study Group. Mult Scler. (1999) 5:78-88. doi: $10.1177 / 135245859900500203$

20. Foster M, Zivadinov R, Weinstock-Guttman B, Tamano-Blanco M, Badgett D, Carl E, et al. Associations of moderate alcohol consumption with clinical and MRI measures in multiple sclerosis. J Neuroimmunol. (2012) 243:61-8. doi: 10.1016/j.jneuroim.2011.12.007

21. Weiland TJ, Hadgkiss EJ, Jelinek GA, Pereira NG, Marck CH, Van Der Meer DM. The association of alcohol consumption and smoking with quality of life, disability and disease activity in an international sample of people with multiple sclerosis. J Neurol Sci. (2014) 336:211-9. doi: 10.1016/j.jns.2013.10.046

22. Beier M, D'orio V, Spat J, Shuman M, Foley FW. Alcohol and substance use in multiple sclerosis. J Neurol Sci. (2014) 338:122-7. doi: 10.1016/j.jns.2013.12.029
23. D’hooghe MB, Haentjens P, Nagels G, De Keyser J. Alcohol, coffee, fish, smoking and disease progression in multiple sclerosis. Eur J Neurol. (2012) 19:616-24. doi: 10.1111/j.1468-1331.2011.03596.x

24. Diaz-Cruz C, Chua AS, Malik MT, Kaplan T, Glanz BI, Egorova S, et al. The effect of alcohol and red wine consumption on clinical and MRI outcomes in multiple sclerosis. Mult Scler Relat Disord. (2017) 17:47-53. doi: 10.1016/j.msard.2017.06.011

25. Bombardier CH, Blake KD, Ehde DM, Gibbons LE, Moore D, Kraft GH. Alcohol and drug abuse among persons with multiple sclerosis. Mult Scler. (2004) 10:35-40. doi: 10.1191/1352458504ms989oa

26. Quesnel S, Feinstein A. Multiple sclerosis and alcohol: a study of problem drinking. Mult Scler. (2004) 10:197-201. doi: 10.1191/1352458504ms992oa

27. Capurso G, Lahner E. The interaction between smoking, alcohol and the gut microbiome. Best Pract Res Clin Gastroenterol. (2017) 31:579-88. doi: 10.1016/j.bpg.2017.10.006

28. Dopkins N, Nagarkatti PS, Nagarkatti M. The role of gut microbiome and associated metabolome in the regulation of neuroinflammation in multiple sclerosis and its implications in attenuating chronic inflammation in other inflammatory and autoimmune disorders. Immunology. (2018) 154:178-85. doi: $10.1111 / \mathrm{imm} .12903$

29. Goral J, Karavitis J, Kovacs EJ. Exposure-dependent effects of ethanol on the innate immune system. Alcohol. (2008) 42:237-47. doi: 10.1016/j.alcohol.2008.02.003

30. Marrie R, Horwitz R, Cutter G, Tyry T, Campagnolo D, Vollmer T. High frequency of adverse health behaviors in multiple sclerosis. Mult Scler. (2009) 15:105-13. doi: $10.1177 / 1352458508096680$

Conflict of Interest Statement: The authors declare that the research was conducted in the absence of any commercial or financial relationships that could be construed as a potential conflict of interest.

The reviewer KM declared a past co-authorship with one of the authors ML to the handling editor.

Copyright $\odot 2019$ Ivashynka, Copetti, Naldi, D'Alfonso and Leone. This is an openaccess article distributed under the terms of the Creative Commons Attribution License (CC BY). The use, distribution or reproduction in other forums is permitted, provided the original author(s) and the copyright owner(s) are credited and that the original publication in this journal is cited, in accordance with accepted academic practice. No use, distribution or reproduction is permitted which does not comply with these terms. 